\title{
Medical student wellness and professional development are harmed by the current state of medical licensing examination: a student perspective on Step 1
}

\author{
Lucas Lebovitz, ${ }^{1}$ Brian $\mathrm{Wu}^{2}$ \\ ${ }^{1}$ Keck School of Medicine of USC; ${ }^{2}$ Psychiatry, LAC+USC Medical Center, Los Angeles, CA, USA
}

\section{Introduction}

The first part of the United States Medical Licensing Examination, also known as Step 1, has stood since its inception in the 1990s as a requirement for prospective doctors to obtain a medical license. For the purposes of licensure, performance on the exam is pass/fail. However, it has also historically been reported as a numerical score, which holds a critical role in applying for residency: it was the most commonly considered factor in an application by program directors in the most recent national survey. ${ }^{1}$ Currently the Invitational Conference on USMLE Scoring (InCUS) is considering changing Step 1 scoring to only reporting a pass or fail. ${ }^{2}$ Many good reasons exist to move to pass/fail: it was never intended to stratify students, it may not predict performance in internship ${ }^{3}$ and test scores show gender and racial differences even after controlling for covariates. ${ }^{4}$ However, one important consideration should be the substantial toll on mental health

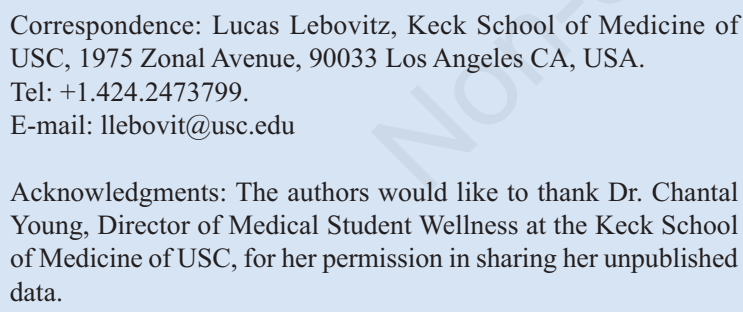
Young, Director of Medical Student Wellness at the Keck School of Medicine of USC, for her permission in sharing her unpublished data.

Key Words: USMLE; medical student; wellness.

Contributions: LL; literature search and manuscript writing; BW; manuscript reviewing.

Received for publication: 25 October 2019.

Accepted for publication: 2 December 2019.

This work is licensed under a Creative Commons Attribution NonCommercial 4.0 License (CC BY-NC 4.0).

${ }^{\circ}$ Copyright: the Author(s), 2019

Licensee PAGEPress, Italy

Qualitative Research in Medicine \& Healthcare 2019; 3:124-125

doi:10.4081/qrmh.2019.8639 and our capacity for professional identity formation that results from numerical Step 1 scores.

In recent years there has been a dramatic increase in awareness, research, and action concerning the mental health of medical students. A recent meta-analysis showed that $27.2 \%$ of medical school students showed evidence of depression or depressive symptoms, and a staggering $11.1 \%$ of students had suicidal ideation. ${ }^{5}$ A different study with over 1000 medical students had a prevalence of anxiety of $25.7 \%$, and $11.7 \%$ of students had suicidal thoughts within the last year. ${ }^{6}$ When medical student suicides do occur, they rock the foundations of their communities and demand cultural and institutional action to address the ways in which medical education contributes to this epidemic. ${ }^{7}$ In its current form the USMLE Step 1 Exam, in addition to its flaws as an objective differentiator of applicants, causes lasting damage to mental health and professional development, and a serious attempt to improve the wellness of medical students must include changes to the exam.

It is not news that Step 1 is a major stressor for medical students, but what may be surprising to some is how much the stress of Step 1 can dwarf other seemingly pressing concerns. A Director of Medical Student Wellness at one institution was consulted about the role of Step 1 as a stressor after having collected pertinent data from hundreds of students. At this institution, when asked to rate stressors from a list of 12 , including the total amount of material that has to be learned, expectations of research activities, performing clinical skills, and loneliness, students rated Step 1 as the greatest stressor across all four years of medical school. More to the point, fully a year before Step 1, first-year medical students at one institution rated it as their single worst stressor. The way students react to this level of stress damages our broader development, but it is valuable to dwell on this point a little longer: first-year medical students should be worrying about other things, such as exploring future specialties, newfound responsibilities for patients, or pursuing research. The fact that a licensing exam over a year away, and only one of the three in medical school alone, stresses out first-year students more than anything else should give medical educators and legislators pause. 
The way students prepare for Step 1 can significantly derange wellness. Students commonly beginning studying for Step 1 years ahead of the exam, and studying intensifies during a period referred to as "dedicated time", when surveys indicate students spend over a month studying for over 11 hours per day. ${ }^{7}$ Studying for Step 1 is isolating and damaging. In a lecture that one author's medical school delivered to warn students of what lay in store, students were advised to tell their loved ones that they might be absent. The competitive outcome of the test even makes it difficult for some students to find commiseration in their peers. Just as many students shut themselves away from friends or family to study, there is also a mass exodus months ahead of the exam from student-run clinics, specialty interest groups, music, sports, and other extracurricular activities. These passions outside of class that made students interesting applicants and buttressed mental health are all jettisoned like ballast. Students are soon to be thrown onto the wards, and these experiences are needed much more than an encyclopedic knowledge of rare vitamin deficiencies to handle the stresses of the years of patient care.

In addition to the damage to mental health, studying for Step 1 hamstrings professional identity development. It is no secret that medical students have increasingly sought outside resources for education, ${ }^{8}$ but Step 1 intensifies the issue. Beginning often up to a year ahead of the exam, tens of thousands of students start to focus on expensive subscriptions to third-party question banks, videos, and review books, sometimes eschewing entirely the schools' curricula. What these review materials give in purified basic science knowledge they lack in everything else that is necessary for a trainee to become a fully-fledged physician: explorations of alternative and integrative medicine, introductions to malpractice law and policy advocating, discussions of social determinants of health, and more. To be clear, this institution and others commit time to this aspect of the curriculum, but during Step 1 studying students are intentionally often physically or mentally absent. What results is a new provider with a highly specialized education, much of which almost irrelevant to patient care. Our future patients clearly don't want a less competent physician, but they should be concerned to see humanistic care sacrificed for ballooning test scores.

These months of myopia may be causing damage to the professional identities of students, but they are a smart group who knows the stakes in this game. Little is more important for induction as doctors than that score, and the necessary steps to become future leaders in our fields, researchers, teachers, policy advocates, and members of the community are simply not tested on Step 1. All students at some point are forced to sacrifice opportunities for growth to score a few points higher. An anonymous second-year medical student exemplifies this attitude in the following quotation, taken from a medical educator reporting student feedback on Twitter:
The issue is Step 1. Step 1 is the difference between having the option to do what I want for the rest of my life and a compromise. Anything that gets in the way of that doesn't seem helpful at the moment. ${ }^{9}$

For decades Step 1 has been the tape with which we measure medical students. Reliance on it by residency directors, for whatever reason, has resulted in an intensely stressful and myopic culture amongst medical students that has real mental health consequences. A pass-fail system for Step 1 has been proposed, albeit with pushback from residency programs looking for objective metrics. ${ }^{10}$ Others imagine a quartile or quintile system which dilutes the negatives of numerical grades. Either solution is better than the status quo. The status of Step 1 grading remains up in the air, and a final direction from INCUS has yet to be announced, so there remains time for physicians, legislators, and medical educators to argue for a more humane, and humanistic, method of education. If the medical community is serious about decreasing physician suicides, we must continue to make institutional-level changes such as these to address the damage caused to medical student wellness and future professional identities.

\section{References}

1. The Match. National Residency Matching Program [Internet]. Results of the 2018 NRMP Program Director Survey. Available from: https:/www.nrmp.org/wp-content/uploads/2018/07/NRMP-2018-Program-Director-Survey-forWWW.pdf

2. Weiner S. Should the USMLE be pass/fail? Association of American Medial Collegers 2019. Available from: https:// www.aamc.org/news-insights/should-usmle-be-pass/fail

3. Lee M, Vermillion M. Comparative values of medical school assessments in the prediction of internship performance. Med Teach 2018;40:1287-92.

4. Rubright JD, Jodoin M, Barone MA. Examining demographics, prior academic performance, and united states medical licensing examination scores. Acad Med 2019;94:364-70.

5. Rotenstein LS, Ramos MA, Torre M, et al. prevalence of depression, depressive symptoms, and suicidal ideation among medical students: a systematic review and meta-analysis. JAMA 2016;316:2214-36.

6. MacLean L, Booza J, Balon R. The impact of medical school on student mental health. Acad Psychiatr 2016;40:89-91.

7. Muller D. Kathryn. N Engl J Med 2017;376:1101-3.

8. Burk-Rafel J, Santen SA, Purkiss J. Study behaviors and USMLE Step 1 performance: implications of a student selfdirected parallel curriculum. Acad Med 2017;92:S67-74.

9. Carmody B. Twitter 2018. Available from: https:// twitter.com/jbcarmody/status/1079183928911638529

10. De Haan JB, Markham T, Ghebremichael S. considerations of a resident recruitment committee on the USMLE Step 1 examination. Acad Med 2019;94:922-3. 